December 2006

\title{
Biomolecular tuning of electronic transport properties of carbon nanotubes via antibody functionalization
}

\author{
Kasif Teker \\ Frostburg State University \\ Dr. Eric Wickstrom \\ Thomas Jefferson University \\ Balaji Panchapakesan \\ University of Delaware
}

Follow this and additional works at: https://jdc.jefferson.edu/bmpfp

Part of the Medical Biochemistry Commons

\section{Let us know how access to this document benefits you}

\section{Recommended Citation}

Teker, Kasif; Wickstrom, Dr. Eric; and Panchapakesan, Balaji, "Biomolecular tuning of electronic transport properties of carbon nanotubes via antibody functionalization" (2006). Department of Biochemistry and Molecular Biology Faculty Papers. Paper 8.

https://jdc.jefferson.edu/bmpfp/8

This Article is brought to you for free and open access by the Jefferson Digital Commons. The Jefferson Digital Commons is a service of Thomas Jefferson University's Center for Teaching and Learning (CTL). The Commons is a showcase for Jefferson books and journals, peer-reviewed scholarly publications, unique historical collections from the University archives, and teaching tools. The Jefferson Digital Commons allows researchers and interested readers anywhere in the world to learn about and keep up to date with Jefferson scholarship. This article has been accepted for inclusion in Department of Biochemistry and Molecular Biology Faculty Papers by an authorized administrator of the Jefferson Digital Commons. For more information, please contact: JeffersonDigitalCommons@jefferson.edu. 


\title{
Biomolecular Tuning of Electronic Transport Properties of Carbon Nanotubes via Antibody Functionalization
}

\author{
Kasif Teker, Eric Wickstrom, and Balaji Panchapakesan
}

\begin{abstract}
Carbon nanotubes (CNTs) are remarkable solidstate nanomaterials due to their unique electrical and mechanical properties. The electronic properties of nanotubes combined with biological molecules such as proteins could make miniature devices for biological sensing applications. In this paper, the noncovalent interaction of single-wall CNTs with antibodies is presented for its potential applications for detecting overexpressed cell surface receptors in breast cancer cells. The degree of binding of antibodies on CNTs was found to be more than $80 \%$ for an extended sampling area by confocal microscopy. The key to achieve such high degree of functionalization is due to the separation of CNTs using surfactants that leads to a high surface area to volume ratio and higher number of active sites for charge transfer that enhance binding. This paper also presents tuning of electronic transport properties of CNTs by monoclonal antibodies that are specific to insulin-like growth factor 1 receptor in breast cancer.
\end{abstract}

Index Terms-Antibody functionalization, breast cancer, carbon nanotube field-effect transistors (CNTFETs), drug delivery, insulin-like growth factor 1 receptor (IGF1R).

\section{INTRODUCTION}

C ARBON nanotubes (CNTs) are novel synthetic materials with unique electronic [1] and mechanical properties [2]. They can be either metallic or semiconducting depending on their diameter and chirality [3], thereby offering possibilities to form metal-semiconductor and semiconductor-semiconductor junctions, useful in electronic and sensor devices. CNTs have found applications as building blocks for nanodevices such

Manuscript received March 15, 2005; revised March 23, 2006 and July 3, 2006. This work was supported by the Department of Defense of the Office of Congressionally Directed Medical Research Program under BCRP Concept Award BC024244 and by the INBRE program of the National Center for Research Resources (NCRR); which is a component of the National Institutes of Health (NIH), under Grant 2 P20 RR016472-06. This work was also supported in part by the National Science Foundation under Career Award ECS:0546328 to one of the authors (B. P.). The associate editor coordinating the review of this paper and approving it for publication was Prof. Michiel Vellekoop.

K. Teker was with the Delaware MEMS and Nanotechnology Laboratory, Department of Electrical and Computer Engineering, University of Delaware, Newark, DE 19716 USA. He is now with the Department of Physics and Engineering, Frostburg State University, Frostburg, MD 21532 USA (e-mail: kteker@frostburg.edu).

E. Wickstrom is with the Department of Biochemistry and Molecular Biology, Thomas Jefferson University, Philadelphia, PA 19107-5587 USA (e-mail: Eric.Wickstrom@jefferson.edu).

B. Panchapakesan is with the Department of Electrical Engineering, University of Delaware, Newark, DE 19716 USA (e-mail: baloo@ece.udel.edu).

Color versions of Figs. 2, 3, 5, and 6 are available online at http://ieeexplore.ieee.org.

Digital Object Identifier 10.1109/JSEN.2006.884564 as transistors [4], probes [5], electron-field emission sources [6], and chemical sensors [7]. In addition, potential biological devices, which are fabricated by integrating nanotubes with organic molecules [8]-[10], will enable new research fields and applications such as in situ modification of living cells or their physiological activities [11].

The research of CNT functionalization has been intensified due to their great potential for biomedical and biotechnological applications. Organic modification of CNTs generates multiple sites for the attachment of bioactive molecules, and the modified nanotube could be used as a biosensor or a novel delivery system. Therefore, understanding the interaction of CNTs with biological systems is essential for the realization of biohybrid systems. In very recent studies, nanotubes have been functionalized to study their biocompatibility and protein recognition capability. Davis et al. [12] reported the immobilization of proteins and enzymes in CNTs. They reported that small proteins and enzymes can be readily placed within the interior cavity of opened nanotubes without any drastic conformational change. Chen et al. [13] demonstrated protein binding to single-wall CNTs (SWNTs) through a noncovalent sidewall functionalization scheme. A variety of smaller proteins, such as streptavidin and ferritin, have been immobilized on SWNTs that were functionalized by 1-pyrene butanoic acid succinimidyl ester. The pyrenel group irreversibly adsorbs onto the hydrophobic surfaces of SWNTs through $\pi-\pi$ interaction, and the succinimidyl ester group reacts with amine groups on lysine residues of proteins to form amide bonds. More recently, Shim et al. [14] investigated the adsorption behavior of the streptavidin/biotin system on SWNTs. They achieved specific binding of streptavidin onto SWNTs by cofunctionalization of nanotubes with biotin and protein-resistant polymers. In another study, CNTs were functionalized by bovine serum albumin (BSA) proteins via diimide-activated amidation [15]. They claimed that the vast majority of the protein species in the nanotube BSA conjugates remained bioactive.

Converting nanotubes into electronic devices for biosensor applications have received significant attention in recent years. The reason for such an approach is to be able to combine nanotechnological interfaces to CMOS, MEMS, and biotechnology that can lead to creation of compact and batch-fabricated biosensors with hundreds of sensors on a single chip. This would be a powerful approach for simultaneously detecting and screening various types of diseases. An example to such device is a nanoscale field-effect transistor (FET) with CNTs 
acting as the conducting channel for detection of various biomolecules. CNT-based FET (CNTFET) has first been fabricated in 1998 [4] and extensively studied [16]. The active detection area of the nanotube-based devices can be sized for individual biomolecules. Furthermore, their response to different species can be varied in a controlled way using chemical and biological functionalization. Advances in the fabrication of the nanotubebased FET devices have raised the expectations for the utilization of CNTs as biosensor materials [17], [18].

Although functionalization of CNTs with smaller proteins (streptavidin $\sim 60 \mathrm{kDa} /$ biotin) has been studied recently [14], it is particularly important to study the interaction of larger antibodies $(\sim 150 \mathrm{kDa})$ on CNTs due to their direct application in biomedical nanotechnology for cancer detection. Antibodies, which are proteins of the class of immunoglobulins (IgGs), are produced to respond to foreign substances called antigens. In fact, antibodies are the most important molecules of the vertebrate immune system. One of the most important characteristics of antibodies is specificity, i.e., each antibody binds to a particular antigen. Furthermore, the antibodies can be used to protect against disease, help diagnose various illnesses, and detect drugs and abnormal substances in the blood. In this paper, the following studies are presented toward developing CNT-based cancer diagnostic systems: 1) separation of CNTs in aqueous solutions, 2) functionalization of CNTs with antibodies that are specific to cancer cell surface receptors, and 3) electronic detection of antibodies using CNTFETs. We have functionalized CNTs with three different antibodies: 1) monoclonal mouse $\operatorname{IgG}, 2$ ) polyclonal rabbit anti-goat IgG, and 3) polyclonal goat-anti-mouse IgG. It should be pointed that BT474 human breast cancer cells overexpress HER2 (Her2+) and c-MYC oncogenes but not the estrogen receptor $\left(\mathrm{ER}^{-}\right)$or insulin-like growth factor 1 receptor $\left(\mathrm{IGF} 1 \mathrm{R}^{-}\right)$with MCF7M human breast cancer cells, which express the estrogen receptor $\left(\mathrm{ER}^{+}\right)$and overexpress cyclin $\mathrm{D} 1$ and c-MYC oncogenes and the IGF1R $\left(\mathrm{IGF}^{+}\right)$[19]-[21]. Therefore, nanotubes are used as probes for detecting cancer cells by assembling specific anti-oncogene antibodies on nanotube surfaces for detecting overexpressed cell surface receptors (IGF1R and HER2).

This paper describes two main aspects of applications of CNTs for cancer research: 1) utilization of CNTs for drug delivery and 2) sensing of monoclonal antibodies (mAb) specific to cell surface antigens overexpressed on cancer cells by CNTFETs. The drug delivery studies have started with the separation of CNTs in physiological solutions. Aqueous solubility of CNTs has significant implications in biochemistry and biomedical engineering, in which organic solvents cannot be used due to their incompatibilities with living cells and organisms. Following solubility studies, the interaction between CNTs and antibodies is presented by confocal microscopy for the first time. Confocal microscopy provides statistical evaluation of the colocalization of the complex samples by combining high-resolution sampling with observation over extended areas. Electronic detection of monoclonal antibodies has been conducted to develop new technologies for reliable early detection of cancer from biological fluids through minimally invasive or noninvasive procedures. Circulating cancer cells often express characteristic cell surface markers, which provide an opportunity for early diagnosis of progressive disease. We suggest that interaction of adsorbed $\mathrm{mAb}$ with breast cancer cell surface targets would produce a change in the electrical conductance of the SWNT devices dependent on the density of those surface markers on breast cancer cells. This change in the electrical conductance of the SWNT devices could lead to detection of live cancer cells from biological fluids through minimally invasive or noninvasive procedures.

\section{EXPERIMENTAL DETAILS}

SWNT solution was prepared in deionized (DI) water with a surfactant, i.e., sodium dodecyl benzene sulfonate (NADDBS: ICN Biomedicals, Inc.), which ensured their separation in an aqueous environment. The entire mixture was gently agitated for $24 \mathrm{~h}$ in a sonicator (Fisher Scientific Ultrasonic Cleaner, $60-\mathrm{Hz}$ frequency, $\mathrm{FS} 60 \mathrm{H}$ ) at room temperature that resulted in separation of the SWNT bundles into individual SWNT. The NADDBS to CNT ratio is 20 by weight [22]. Following that, the well-separated nanotubes were labeled with dihexyloxacarbocyanine iodide $\left(\mathrm{DiOC}_{6}\right.$ : Molecular Probes, Inc.), which is a dye that fluoresces green when excited with argon laser at $488 \mathrm{~nm}$. The $\mathrm{DiOC}_{6}$ was prepared at $2 \mathrm{mg} / \mathrm{ml}$ in methanol and diluted in DI water just prior to use with the CNT solution. The dye and nanotube solution were mixed in a microcentrifuge tube at a $1: 1$ ratio and allowed to interact for $1 \mathrm{~h}$.

The antibody solutions were prepared in phosphate-buffered saline (PBS) (0.138 M NaCl, $0.0027 \mathrm{M} \mathrm{KCl}, \mathrm{pH} 7.4)$ by diluting a $2 \mathrm{mg} / \mathrm{ml}$ antibody solution with PBS to a ratio of $1: 10$ (antibody/PBS). Then, the antibody solutions were prelabeled with Alexa 546 (Molecular Probes, Inc.), which is a dye that fluoresces red when excited with $\mathrm{HeNe}$ laser at $543 \mathrm{~nm}$. The dye-labeled CNT solution and the prelabeled antibody solution were mixed in a microcentrifuge tube and allowed to interact for up to $2 \mathrm{~h}$ prior to confocal microscopy analysis. Centrifuging was done to the antibody solution, when necessary, to eliminate unnecessary fluorescence. A slightly different procedure was carried out to view the monoclonal mouse $\operatorname{IgG}$ antibodies by confocal microscopy since the presence of BSA with the antibody interferes the labeling of the antibodies. Therefore, the unlabeled mouse antibody was first tagged with the fluorescently labeled polyclonal goat anti-mouse antibody, which selectively binds to the monoclonal mouse antibody. Then, the monoclonal mouse and polyclonal goat anti-mouse antibody conjugate was introduced to the dye-labeled CNT solution. Besides confocal microscopy, scanning electron microscopy (SEM) and atomic force microscopy (AFM) were used for analysis. Confocal microscope is an enhanced fluorescent microscope where outof-focus fluorescent light is eliminated by using a confocal aperture (pinhole). Confocal microscopy provides observation of spatially extended areas as well as high-resolution analysis. The advantage of fluorescence for microscopy is the possibility to analyze location and expression of many target molecules at the same time. Furthermore, a full three-dimensional view of the sample can be obtained by integrating the optically sectioned thin slices almost in real time. Moreover, confocal microscopy provides statistical evaluation of the colocalization 


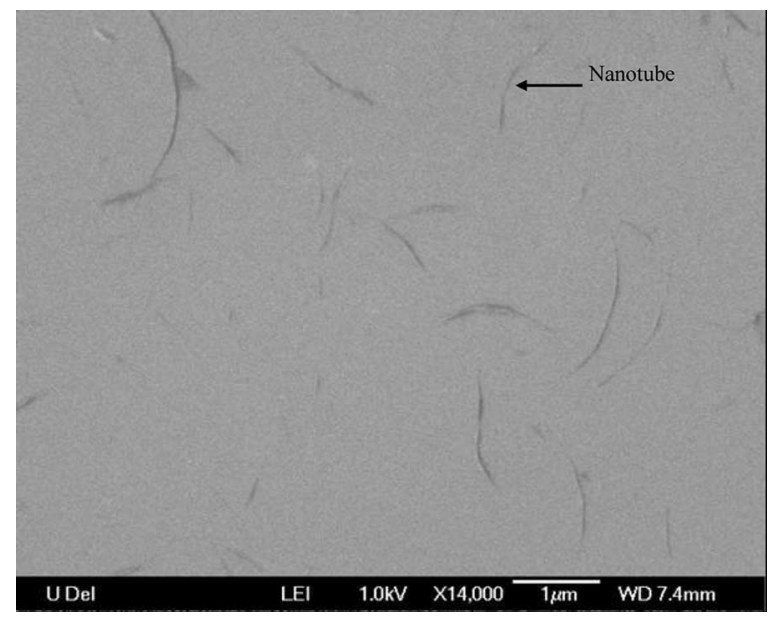

Fig. 1. SEM image of the separated CNTs in water with the surfactant NaDDBS. The CNTs are very well separated and dispersed.

of the complex samples by combining high-resolution sampling with observation over extended areas. Therefore, we have used confocal microscopy to view fluorescently tagged CNTs in a method similar to that of viewing biological materials such as antibodies to accurately analyze and quantify their interaction. Electrical measurements were performed using CNTFETs fabricated with $\mathrm{Ti} / \mathrm{Au}(10: 100 \mathrm{~nm})$ electrodes on a Signatone S1160 probe station attached to HP 8466 Semiconductor Parameter Analyzer to detect the change in conductance of the nanotube-based FET devices due to antibody incubation.

\section{RESUlTS AND DiscUSSIONS}

\section{A. Antibody Functionalization of CNTs}

Aqueous solubility of CNTs has significant implications in biochemistry and biomedical engineering, in which organic solvents cannot be used due to their incompatibilities with living cells and organisms. In addition, it is very critical to preserve electronic properties of CNTs following the solubility treatments. Therefore, noncovalent stabilization of CNTs using surfactants has become an attractive approach for nanotube dispersion and separation. It was reported that surfactant molecules adsorb in between the gaps of a nanotube bundle and eventually separate the individual nanotubes from the bundle [23]. Fig. 1 is an SEM image of CNTs showing a very effective separation of CNT bundles into individual CNTs using the surfactant NaDDBS. This is clearly one of the most important steps to investigate functionalization of antibodies on nanotube surfaces due to achieving very high surface area to volume ratio. The NaDDBS has the ability to break up the CNT bundles into individual nanotubes without forming chemical bonds. It is believed that $\pi$-like stacking of the benzene rings onto the graphite surfaces enhances the binding and surface coverage of surfactant molecules to graphite [24]. It has been reported that during the adsorption of the surfactant NaDDBS on SWNTs, Coulombic forces do not play a central role but are overcome by the hydrophobic interactions between the surfactant tail and the nanotube walls [25]. Each nanotube is covered by a monolayer of surfactant molecules in which the tails remain in contact with the nanotube walls, whereas the heads form a compact outer surface.

The well-separated nanotubes were then labeled by $\mathrm{DiOC}_{6}$ due to the fact that CNTs are too small to observe without the aid of fluorescent tags. Although it may be possible to image the larger CNT bundles with phase or differential interference contrast imaging with the help of video enhancement, fluorescence labeling allows individual small CNTs and attached molecules to be imaged with confocal microscopy. Furthermore, labeling CNTs with conventional fluorophores makes the observation and manipulation of CNTs and organic materials in physiological solutions very facile compared to electron microscopic techniques. Moreover, nanotubes coated with fluorophores can be used for fabricating biological nanoprobes in cytological research.

Noncovalent attachment of $\mathrm{DiOC}_{6}$ to the CNTs allows imaging the entire CNTs without altering $\mathrm{sp}^{2}$-bonded graphene sidewall. It was reported that the noncovalent attachment of the dye to the CNTs could occur through hydrophobic interactions [26]. It is believed that the binding was mediated through the hydrocarbon chains. Long hydrophobic hydrocarbon chains of the $\mathrm{DiOC}_{6}$ would allow stable binding with the CNTs. Furthermore, the separation of the CNTs significantly enhanced the labeling efficiency of the CNTs by $\mathrm{DiOC}_{6}$.

Having successfully achieved the separation and labeling of CNTs, the antibody functionalization of the CNTs has been carried out. Fig. 2(a) is an overlap confocal image of CNTs (green) and antibodies (red). The green dye in the inner rectangular region is bleached out to verify the attachment of the antibodies to the CNTs. To evaluate the degree of binding over extended areas, weighted colocalization coefficients (WCCs) for both the CNTs and antibodies were calculated. WCC can be defined as the ratio of the intensity of colocalized area of a particular channel (color) to the intensity of total area above threshold intensity of that channel (color). It is known that the colocalization coefficients can provide quantitative information in dualcolor images [27], and the same procedure was followed here for antibody functionalization. Fig. 2(b) is a scattergram that provides quantitative information about the image. The WCCs were found to be more than 0.80 for both the CNTs and antibodies. To determine the repeatability, six consecutive experiments have been conducted under the same conditions. The calculations yielded high degree of colocalization with an average WCC of 0.80 for CNTs and 0.87 for antibodies [28]. The key to such high WCC is due to the separation of CNTs using a surfactant solution. When nanotubes are separated using a surfactant, it yields high surface area that enables attachment of antibodies on the nanotube surface without affecting the sidewall, thereby yielding high WCC. Fig. 3 is an AFM image showing the attachment of the antibodies to a single nanotube. Furthermore, AFM height measurements were performed. It was found that the surfactant-coated nanotube is about $5 \mathrm{~nm}$ high. The height of the surfactant- and antibody-coated nanotube is $16 \mathrm{~nm}$.

\section{B. Antibody Sensing via CNTFETs}

CNTFET devices have been fabricated on a doped silicon wafer that is coated with silicon dioxide. First, metal 


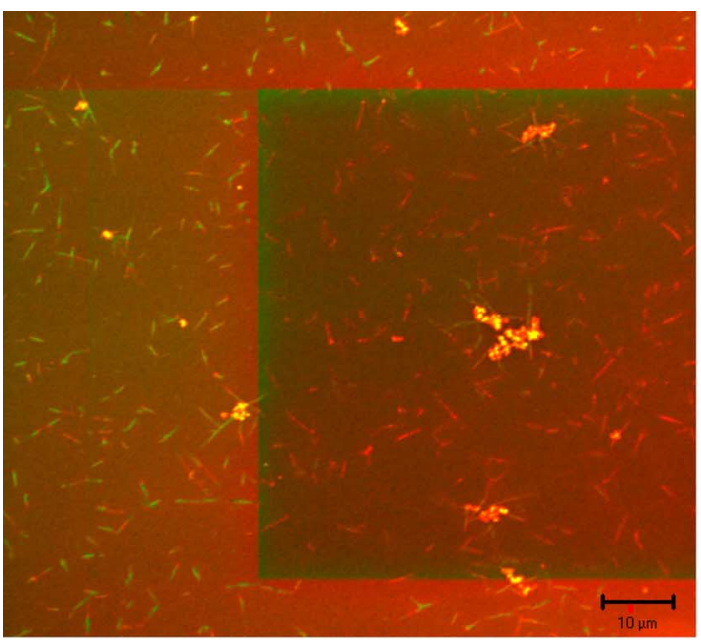

(a)

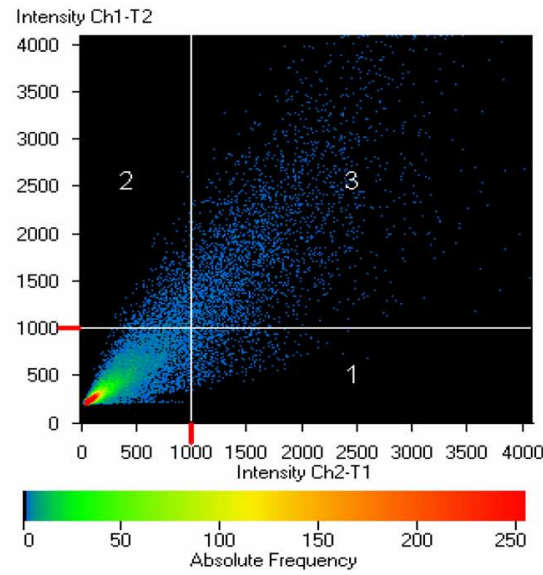

Fig. 2. (a) Confocal microscopy image of green-dye-labeled CNTs and subsequently coated with the red-dye-conjugated antibodies The green dye (labeling CNTs) in the inner rectangular region is bleached to verify the selective attachment of the antibodies to the CNTs (i.e., CNTs appear in red color due to the antibody binding). (b) Scattergram to measure the colocalization between antibodies and CNTs via calculation of WCC.

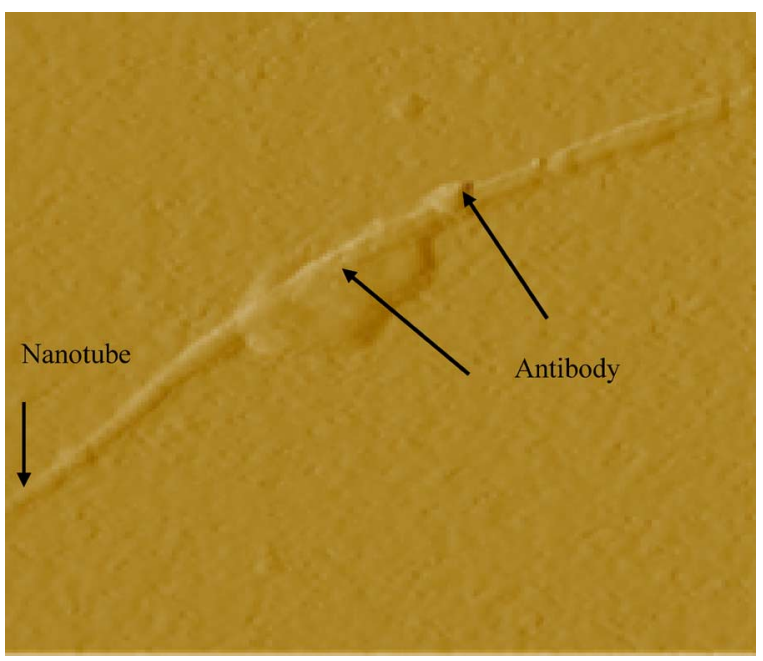

Fig. 3. AFM image of the same sample Fig. 2(a) showing the attachment of the antibodies to a single CNT. The entire scan size is $1.25 \times 1.25 \mu \mathrm{m}$.

electrodes were patterned using microfabrication techniques such as photolithography, metal evaporation, and liftoff. The electrodes consist of 10-nm titanium and 100-nm gold with

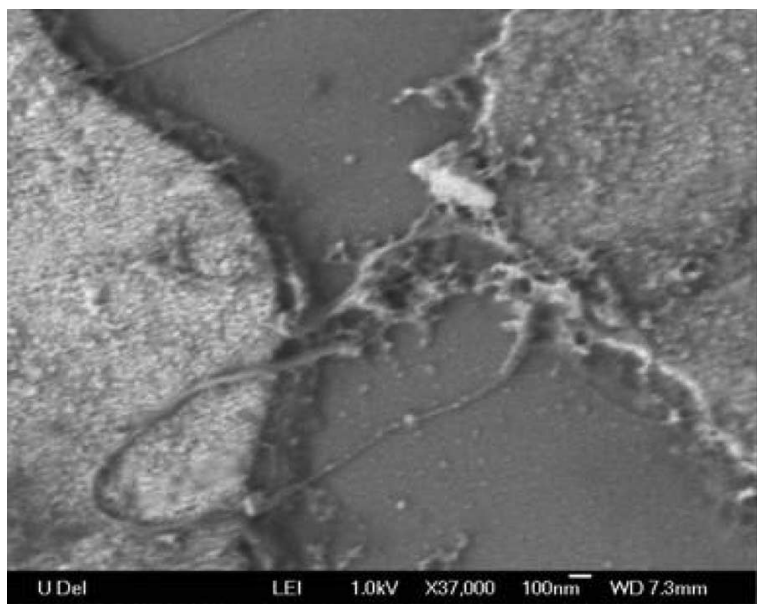

Fig. 4. SEM image of the nanotube FET device.

a spacing of 1-4 $\mu \mathrm{m}$ between source and drain. Following that, the nanotubes were integrated between the electrodes by electrophoretic self-assembly technique. This process has been carried out using ac electrophoresis with a frequency of $10 \mathrm{MHz}$ and voltages of $0.2-1.0 \mathrm{~V}$ peak to peak. Fig. 4 is an SEM image of the nanotube FET device. Following the electrophoretic selfassembly, the devices were dried, and electrical measurements were performed using a probe station attached to a semiconductor parameter analyzer. The silicon substrate was used as back gate. After the measurements, the devices were annealed at $180{ }^{\circ} \mathrm{C}$ for $15 \mathrm{~min}$ to reduce contact resistance between the nanotube and the electrodes. In fact, the resistance of the device dropped from 289 to $84 \mathrm{k} \Omega$. Since SWNTs are a mixture of semiconducting and metallic, OFF-state current in those devices has been observed. The conductance in the p-type region is larger than that in the n-type region because of the work function of the Au electrodes. The Au Fermi level aligns with the valence band of the SWNT, making it p-type contact with a barrier for the injection of electrons. The increase in the conductance of the device could be attributed to the raising of the p-doping level of the device due to $\mathrm{O}_{2}$ exposure during annealing. It was reported that conductance of the CNTFET device can be changed by simple annealing process in various gas ambient [29], [30]. It was also demonstrated that brief annealing of the electrodes at $180{ }^{\circ} \mathrm{C}$ improves the reproducibility of the contact resistance [31]. Furthermore, the device exhibited significant gate effect as determined by $I-V_{G}$ measurements [Fig. 5(a)]. The source-drain current of the devices decreased with increasing gate voltage, indicating that the dominant conduction process is due to hole transport, i.e., p-type semiconducting SWNTs. The conductance of semiconducting SWNTs could be modulated by gate voltages, whereas the conductance of metallic SWNTs is insensitive to the gate voltages. Two features, i.e., gate modulation and high sensitivity to its environment, of semiconducting nanotubes make them excellent candidates for chemical and biosensor applications. The conductance of the SWNT device is sensitive to the surface charge. Thus, these devices could be used to detect antibody-antigen interactions as well as various proteins on cell surfaces by monitoring the corresponding conductance change. 


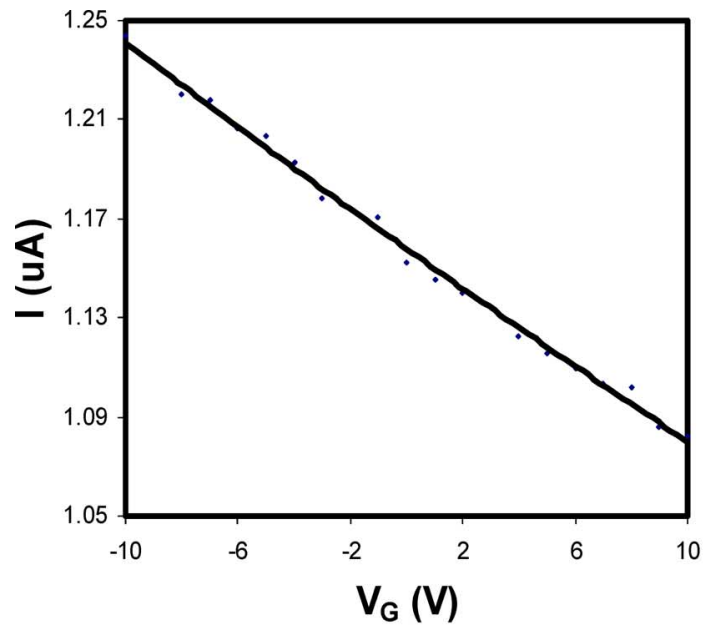

(a)

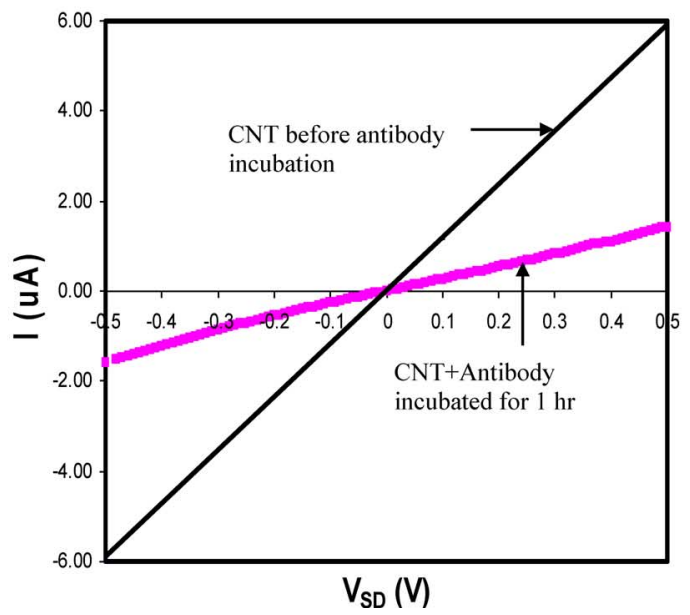

(b)

Fig. 5. Output characteristics of SWNT-FET. (a) Gate voltage dependence of $I_{\mathrm{SD}}$ at $V_{\mathrm{SD}}=100 \mathrm{mV}$ showing gate modulation. (b) Change in device characteristic $I-V_{\mathrm{SD}}$ at $V_{G}=0$ upon exposure to antibody for an hour. The source-drain current was significantly reduced by antibody incubation.

Antibodies were adsorbed on surface of SWNTs. A 2- $\mu$ l monoclonal antibody solution, prepared in PBS and DI water (3.35 $\mu \mathrm{M})$, was adsorbed on the surface of the nanotube. The nanotube and antibodies were allowed to incubate for an hour. The sample was then thoroughly rinsed with DI water. Fig. 5(b) compares the output characteristics $I-V_{\mathrm{SD}}$ of the same device after antibody incubation at zero gate voltage. It should be emphasized that a significant loss of source-drain current was observed upon exposure to antibody. Fig. 6 presents the change in conductance with various antibody concentrations. As the antibody concentration increases, the conductance decreases considerably. The decrease in conductance results from the electron-donating property of the $\mathrm{NH}_{2}$ groups of the antibodies to nanotubes similar to the previous reports in literature [32]-[35]. The measurements have exhibited that 0.04 electrons per adsorbed amine are donated to nanotubes [33], [34]. Thus, this electron-donating effect will result in a reduction in the number of majority carriers (holes) in semiconducting nanotube, thereby reducing the source-drain current. These results clearly exhibit the biomolecular tuning of electronic transport properties of CNTs by antibody adsorption.

To date, the mechanism of nonspecific adsorption of proteins onto the nanotube surfaces is not known. Hydrophobic

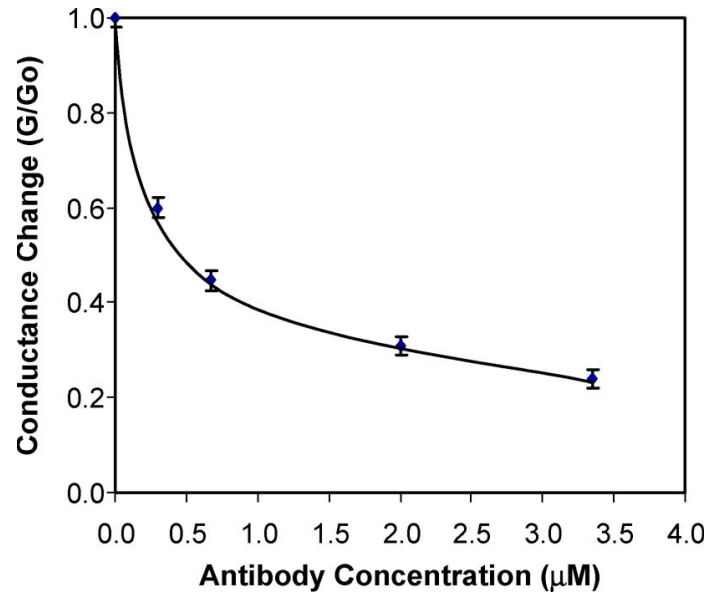

Fig. 6. Change in conductance of SWNT-FET with various antibody concentrations indicating the antibody-induced conductance decrease.

interaction has been the most widely accepted mechanism for nonspecific adsorption of proteins onto the nanotube surfaces. However, it was reported that a highly hydrophobic protein, i.e., fibrinogen, did not adsorb onto SWNTs [14]. In another study, both positively and negatively charged proteins exhibited strong adsorption to SWNTs [36]. This implies that electrostatic interaction mechanism alone could not describe the interaction. In a recent study, an in situ detection of nonspecific adsorption of cytochrome $c$ onto semiconducting SWNT has been investigated by monitoring the conductance change in the nanotube [37]. The protein-induced conductance change was attributed to the positively charged nature of the proteins. Nevertheless, similar study has shown that both positively and negatively charged proteins have exhibited a decrease in conductance [18]. It is evident from these investigations that more studies at the molecular level are required to understand the nonspecific protein-CNT interactions. Nevertheless, the current knowledge on nonspecific protein-nanotube interactions is mature enough to apply for the development of biosensors. Our studies suggest that nanotube-based FET devices can be utilized for electronic sensing of antibodies using CNTs.

\section{CONCLUSION}

We have successfully demonstrated antibody functionalization of CNTs. The CNT bundles were separated to individual nanotubes in aqueous solutions by the surfactant-mediated dispersion method. Following that, the individual CNTs were functionalized with antibodies. A high degree of binding and colocalization (more than 80\%) was observed for an extended sampling area by confocal microscopy. The key to achieving such a high degree of functionalization is due to the separation of CNTs using surfactants, which leads to a high surface area to volume ratio and a higher number of active sites for charge transfer that enhances binding. Although the nonspecific adsorption mechanism of the antibody-nanotube interaction is not well understood, it is believed that the observed high degree of antibody adsorption could be associated with the amino affinity of CNTs. Change in electronic transport properties of semiconducting nanotubes by antibody adsorption has been 
investigated. It was demonstrated that a substantial decrease in conductance was obtained upon antibody adsorption on nanotube surfaces. These results suggest that electronic transport properties of semiconducting nanotubes can be tuned by antibody adsorption. It should also be mentioned that the antibodycoated nanotubes could be used as probes of cell or membrane function due to their smaller size compared to present capillary intracellular probes.

\section{ACKNOWLEDGMENT}

The authors would like to thank Dr. K. Czymmek (Delaware Biotechnology Institute) for his help and useful discussions about confocal microscopy.

\section{REFERENCES}

[1] M. Bockrath, D. H. Cobden, P. L. McEuen, N. G. Chopra, A. Zettl, A. Thess, and R. E. Smalley, "Single-electron transport in ropes of carbon nanotubes," Science, vol. 275, no. 5308, pp. 1922-1925, Mar. 1997.

[2] R. S. Ruoff and D. C. Lorents, "Mechanical and thermal properties of carbon nanotubes," Carbon, vol. 33, no. 7, pp. 925-930, 1995.

[3] P. Avouris, "Carbon nanotube electronics," Chem. Phys., vol. 281, no. 2-3, p. 429, 2002.

[4] R. Martel, T. Schmidt, H. R. Shea, T. Hertel, and P. Avouris, "Singleand multi-wall carbon nanotube field-effect transistors," Appl. Phys. Lett., vol. 73 , no. 17, pp. 2447-2449, Oct. 1998.

[5] H. Dai, J. H. Hafner, A. G. Rinzler, D. T. Colbert, and R. E. Smalley, "Nanotubes as nanoprobes in scanning probe microscopy," Nature, vol. 384 , no. 6605 , p. 147 , Nov. 1996.

[6] W. A. De Heer, A. Chatelain, and D. Ugarte, "A carbon nanotube fieldemission electron source," Science, vol. 270, no. 5239, pp. 1179-1180, Nov. 1995.

[7] J. Kong, N. R. Franklin, C. Zhou, M. G. Chapline, S. Peng, K. Cho, and H. Dai, "Nanotube molecular wires as chemical sensors," Science, vol. 287, no. 5453, pp. 622-625, Jan. 2000.

[8] S. S. Wong, E. Joselevich, A. Woolley, C. L. Cheung, and C. M. Lieber, "Covalently functionalized nanotubes as nanometer-sized probes in chemistry and biology," Nature, vol. 394, no. 6688, p. 52, Jul. 1998.

[9] A. Koshino, M. Yudasaka, M. Zhang, and S. Iijima, "A simple way to chemically react single wall carbon nanotubes with organic materials using ultrasonication," Nano. Lett., vol. 1, no. 7, pp. 361-363, 2001.

[10] V. Georgakilas, K. Kordatos, M. Prato, D. M. Guldi, M. Holzinger, and A. Hirsch, "Organic functionalization of carbon nanotubes," J. Amer. Chem. Soc., vol. 124, no. 5, pp. 760-761, Feb. 2002.

[11] K. Otobe, H. Nakao, H. Hayashi, F. Nihey, M. Yudasaka, and S. Iijima, "Fluorescence visualization of carbon nanotubes by modification with silicon-based polymer," Nano. Lett., vol. 2, no. 10, pp. 1157-1160, 2002.

[12] J. J. Davis et al., "The immobilization of proteins in carbon nanotubes," Inorg. Chem. Acta, vol. 272, no. 1-2, pp. 261-266, 1998.

[13] R. J. Chen, Y. Zhang, D. Wang, and H. Dai, "Noncovalent sidewall functionalization of single-walled carbon nanotubes for protein immobilization," J. Amer. Chem. Soc., vol. 123, no. 16, pp. 3838-3839, Apr. 2001.

[14] M. Shim, N. W. S. Kam, R. J. Chen, Y. Li, and H. Dai, "Functionalization of carbon nanotubes for biocompatibility and biomolecular recognition," Nano Lett., vol. 2, no. 4, pp. 285-288, 2002.

[15] W. Huang et al., "Attaching proteins to carbon nanotubes via diimideactivated amidation," Nano Lett., vol. 2, no. 4, pp. 311-314, Apr. 2002.

[16] A. Bachtold, P. Hadley, T. Nakanishi, and C. Dekker, "Logic circuits with carbon nanotube transistors," Science, vol. 294, no. 5545, pp. 1317-1320, Nov. 2001.

[17] A. Star, J. C. P. Gabriel, K. Bradley, and G. Gruner, "Electronic detection of specific protein binding using nanotube FET devices," Nano. Lett., vol. 3, no. 4, pp. 459-463, 2003.

[18] R. J. Chen, R. S. Bangsaruntip, K. A. Drouvalakis, N. W. S. Kim, M. Shim, Y. Li, W. Kim, P. J. Utz, and H. Dai, "Noncovalent functionalization of carbon nanotubes for highly specific electronic biosensors," Proc. Nat. Acad. Sci. USA, vol. 100, no. 9, pp. 4984-4989, Apr. 2003.

[19] D. F. Hayes, T. M. Walker et al., "Monitoring expression of HER-2 on circulating epithelial cells in patients with advanced breast cancer," Int. J. Oncol., vol. 21, no. 5, pp. 1111-1117, Nov. 2002.
[20] M. A. Guvakova and E. Surmacz, "Overexpressed IGF-I receptors reduce estrogen growth requirements, enhance survival, and promote E-cadherinmediated cell-cell adhesion in human breast cancer cells," Exp. Cell. Res., vol. 231, no. 1, pp. 149-162, Feb. 1997.

[21] X. Le Roy, C. Escot et al., "Decrease of c-erbB-2 and c-myc RNA levels in tamoxifen-treated breast cancer," Oncogene, vol. 6, no. 3, pp. 431-437, Mar. 1991

[22] M. F. Islam, E. Rojas, D. M. Bergey, A. T. Johnson, and A. G. Yodh, "High weight fraction surfactant solubilization of single-wall carbon nanotubes in water," Nano Lett., vol. 3, no. 2, pp. 269-273, Mar 2003.

[23] V. C. Moore, M. S. Strano, E. H. Haroz, R. H. Hauge, and R. E. Smalley, "Individually suspended single-walled carbon nanotubes in various surfactants," Nano Lett., vol. 3, no. 10, pp. 1379-1382, 2003.

[24] J. Liu and W. A. Ducker, "Self-assembled supramolecular structures of charged polymers at the graphite/liquid interface," Langmuir, vol. 16, no. 7, pp. 3467-3473, 2000.

[25] O. Matarredona et al., "Dispersion of single-walled carbon nanotubes in aqueous solutions of the anionic surfactant NADDBS," J. Phys. Chem., B, vol. 107, no. 48, pp. 13357-13367, 2003.

[26] R. Prakash, S. Washburn, R. Superfine, R. E. Cheney, and M. R. Falvo, "Visualization of individual carbon nanotubes with fluorescence microscopy using conventional fluorophores," Appl. Phys. Lett., vol. 83, no. 6, pp. 1219-1221, Aug. 2003.

[27] E. M. M. Manders, F. J. Verbeek, and J. A. Aten, "Measurement of colocalization of objects in dual color confocal images," J. Microsc., vol. 169 , no. 3, pp. 375-382, 1993.

[28] K. Teker, R. Sirdeshmukh, and B. Panchapakesan, "Antibody functionalization of carbon nanotubes for breast cancer applications," in Proc. IEEE Sensors, Vienna, Austria, Oct. 2004

[29] V. Derycke, R. Martel, J. Appenzeller, and P. Avouris, "Carbon nanotube interand intramolecular logic gates," Nano Lett., vol. 1, no. 9, pp. 453-456, 2001.

[30] R. Martel, V. Derycke, C. Lavoie, J. Appenzeller, K. K. Chan, J. Tersoff, and P. Avouris, "Ambipolar electrical transport in semiconducting singlewall carbon nanotubes," Phys. Rev. Lett., vol. 87, no. 25, p. 256805, Dec. 2001.

[31] Z. Yao, C. L. Kane, and C. Dekker, "High-field electrical transport in single- wall carbon nanotubes," Phys. Rev. Lett., vol. 84, no. 13, pp. 2941-2944, Mar. 2000.

[32] K. Bradley, M. Briman, A. Star, and G. Gruner, "Charge transfer from adsorbed proteins," Nano Lett., vol. 4, no. 2, p. 253, 2004

[33] K. Bradley, J. C. P. Gabriel, M. Briman, A. Star, and G. Gruner, "Charge transfer from ammonia physisorbed on nanotubes," Phys. Rev. Lett., vol. 91 , no. 21, p. 218301, Nov. 2003.

[34] H. Chang, J. D. Lee, S. M. Lee, and Y. H. Lee, "Adsorption of $\mathrm{NH}_{3}$ and $\mathrm{NO}_{2}$ molecules on carbon nanotubes," Appl. Phys. Lett., vol. 79, no. 23, pp. 3863-3865, Dec. 2001

[35] J. Kong and H. Dai, "Full and modulated chemical gating of individual carbon nanotubes by organic amine compounds," J. Phys. Chem., B, vol. 105, no. 15, pp. 2890-2893, 2001.

[36] B. R. Azamian, J. J. Davis, K. S. Coleman, C. B. Bagshaw, and M. L. H. Green, "Bioelectrochemical single-walled carbon nanotubes," J. Amer. Chem. Soc., vol. 124, no. 43, p. 12664, 2002.

[37] S. Boussaad, N. J. Tao, R. Zhang, T. Hopson, and L. A. Nagahara, "In situ detection of cytochrome $\mathrm{c}$ adsorption with single walled carbon nanotube device," Chem. Commun., no. 13, p. 1502, 2003.

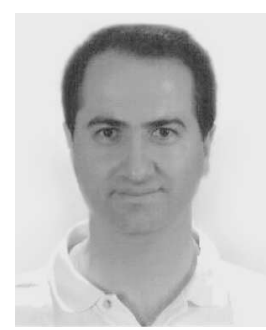

Kasif Teker received the M.S. degree in materials science from Ohio State University, Columbus, in 1996 and the Ph.D. degree in materials science from Case Western Reserve University, Cleveland, OH, in 2001 .

Since 2005, he has been a Faculty Member with the Department of Physics and Engineering, Frostburg State University, Frostburg, MD. As a Postdoctoral Fellow with the University of Delaware, he discovered "single-wall carbon nanotube nanobomb agents for killing breast cancer cells." While a Member of the Technical Staff with IQE Inc., he developed a manufacturable growth technology for indium phosphide-based heterojunction bipolar transistors by molecular beam epitaxy. His current research interests include synthesis of carbon nanotubes, nanotube sensors, cancer nanotechnology, and compound semiconductor heterostructures. 


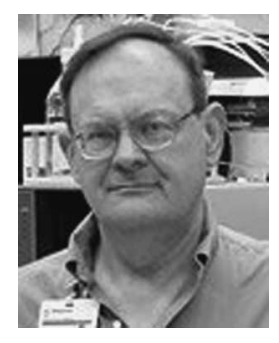

Eric Wickstrom received the B.S. degree (with honors) in biology from California Institute of Technology, Pasadena, and the Ph.D. degree in chemistry from the University of California, Berkeley.

$\mathrm{He}$ is a Professor of biochemistry and molecular biology and a member of the Kimmel Cancer Center, Thomas Jefferson University, Philadelphia, PA. He has published 128 research papers, patents, reviews, chapters, and books on nucleic acid and protein synthesis, structure, and function in cells and animals. His research program develops novel oligonucleotide derivatives for imaging, diagnosis, and therapy of active cancer genes, as well as antibiotics covalently bonded to medical implants.

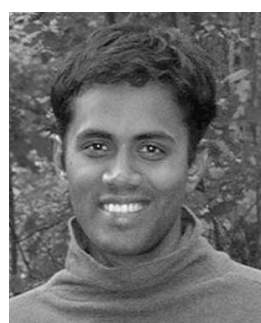

Balaji Panchapakesan received the Ph.D. degree in mechanical engineering from the University of Maryland, College Park, in 2001

Since 2001, he has been an Assistant Professor with the Department of Electrical and Computer Engineering, University of Delaware, Newark. His research interests include fabrication and characterization of carbon nanotubes, antibody functionalization, nanodevices based on carbon nanotubes, and microoptomechanical systems.

Dr. Panchapakesan was the recipient of the National Science Foundation Career Award in 2005. 